\title{
Reaktionen von Derivaten der (Diphenylmethylen-amino)-essigsäure mit Schwefelkohlenstoff und Phenylisothiocyanat sowie Kristall- und Molekülstruktur des 2-(Diphenylmethylen-amino)-2-(1,3-dithian-2-yliden)-essigsäuremethylesters
}

\author{
Reactions of the Derivatives of (Diphenylmethylene-amino) Acetic Acid with Carbon Disulfide \\ and Phenyl Isothiocyanate and Crystal and Molecular Structure of Methyl \\ 2-(Diphenylmethylene-amino)-2-(1,3-dithiane-2-ylidene) Acetate \\ Wolfgang Dölling ${ }^{\mathrm{a}, *}$, Klaus Frost ${ }^{\mathrm{a}}$, Frank Heinemann ${ }^{\mathrm{b}}$ und Helmut Hartung ${ }^{\mathrm{b}}$ \\ ${ }^{a}$ Fachbereich Chemie, Institut für Organische Chemie, b Institut für Physikalische Chemie \\ der Martin-Luther-Universität Halle-Wittenberg, Postfach, D-O-4010 Halle/Saale \\ Herrn Prof. Dr. Manfred Augustin zum 60. Geburtstag gewidmet \\ Z. Naturforsch. 48 b, $493-504$ (1993); eingegangen am 6. November 1992 \\ Alkyl(diphenylmethylene-amino) Acetates, Carbon Disulfide, Ketene Dithioacetals, \\ Alkyl(diphenylmethylene-amino) Acrylates, (Diphenylmethylene-amino) Acrylonitriles \\ Derivatives of (diphenylmethylene-amino) acetic acid 1-3 react in the presence of bases \\ $(\mathrm{NaH}, t-\mathrm{BuONa})$ or under phase transfer conditions with carbon disulfide to give the corre- \\ sponding ketene dithioacetals 4-11 after alkylation. Phenyl isothiocyanate and dicyclohexyl \\ carbodiimide give rise to ketene-S,N- and N,N-acetals. Methyl 2-(diphenylmethyleneamino)- \\ 2-(1,3-dithiane-2-ylidene) acetate (7) was characterized by an X-ray structure determination. \\ The compound has a 2-aza-1,3-butadiene fragment containing two well localized double \\ bonds. The $\mathrm{C}=\mathrm{N}-\mathrm{C}=\mathrm{C}$ group is significantly non-planar, the relevant torsion angle amounts \\ to $-103.5(3)^{\circ}$
}

\section{Einleitung}

Obwohl Reaktionen von Kohlenstoffnucleophilen mit Heterokumulenen zahlreich untersucht wurden, fällt auf, daß Reaktionen von substituierten Aminocarbanionen, im Vergleich zu anderen heterosubstituierten Carbanionen, relativ wenig bearbeitet worden sind. Im Rahmen dieser Arbeit sollen Reaktionen solcher methylenaktiver Verbindungen betrachtet werden, die neben einer Akzeptorgruppe eine Diphenylmethylenimino-Gruppe an der Methylengruppe besitzen. Die Verbindungen 1-3 wurden in diesem Zusammenhang als Ausgangsbasis verwendet. Unter basischen Bedingungen (Deprotonierung) bilden sie $\alpha$-Stickstoffcarbanionen, welche zu Folgereaktionen mit Heterokumulenen wie Schwefelkohlenstoff, Phenylisothiocyanat oder Dicyclohexylcarbodiimid (DCC) befähigt sein sollten.

Jedoch ist zu berücksichtigen, daß sich die speziell aus $\mathbf{1}$ und $\mathbf{2}$ bildenden Esterenolate nicht nur am C-Atom, sondern auch am O-Atom umsetzen lassen sollten, wie kürzlich im Falle von Carbon-

\footnotetext{
* Sonderdruckanforderungen an Dr. habil. W. Dölling.

Verlag der Zeitschrift für Naturforschung,

D-W-7400 Tübingen

0932-0776/93/0400-0493/\$01.00/0
}

säureestern, die nur ein $\alpha$-H-Atom besitzen, gefunden worden ist. Danach lassen sich O-(1-Alkoxy2,2-dialkyl)vinyl-S-alkyl-dithiokohlensäure-diester gewinnen [1,2].

Mit einer Akzeptorwirkung der Diphenylmethylenamino-Gruppe, als zweiter Substituent an der Methylengruppe, ist weniger oder kaum zu rechnen.

Diphenylmethylenamino-substituierte Essigsäure-Derivate werden nach Methoden von O'Donnell [3-6] erhalten.

$\mathrm{Zu}$ Umsetzungen von 1-3 mit Heterokumulenen ist nichts bekannt, jedoch sind Thioacylierungen und Dithiocarboxylierungen von Benzylidenaminoacetonitril beschrieben worden [7-9].

\section{Ergebnisse und Diskussion}

Die Umsetzungen von 1-3 mit Schwefelkohlenstoff erfolgen in DMF unter Verwendung von 2 Äquivalenten Natriumhydrid als Base. Dabei entstehen geminale Dithiolate, die zu den entsprechenden Keten-S,S-acetalen alkyliert werden konnten (s. Schema 1). Die Verbindungen 10 und 11 wurden auf phasentransfer-katalytischem Weg synthetisiert. Als Alkylierungsmittel wurden zwei Äquivalente Methyliodid bzw. 1 Äquivalent $\alpha, \omega$ - 

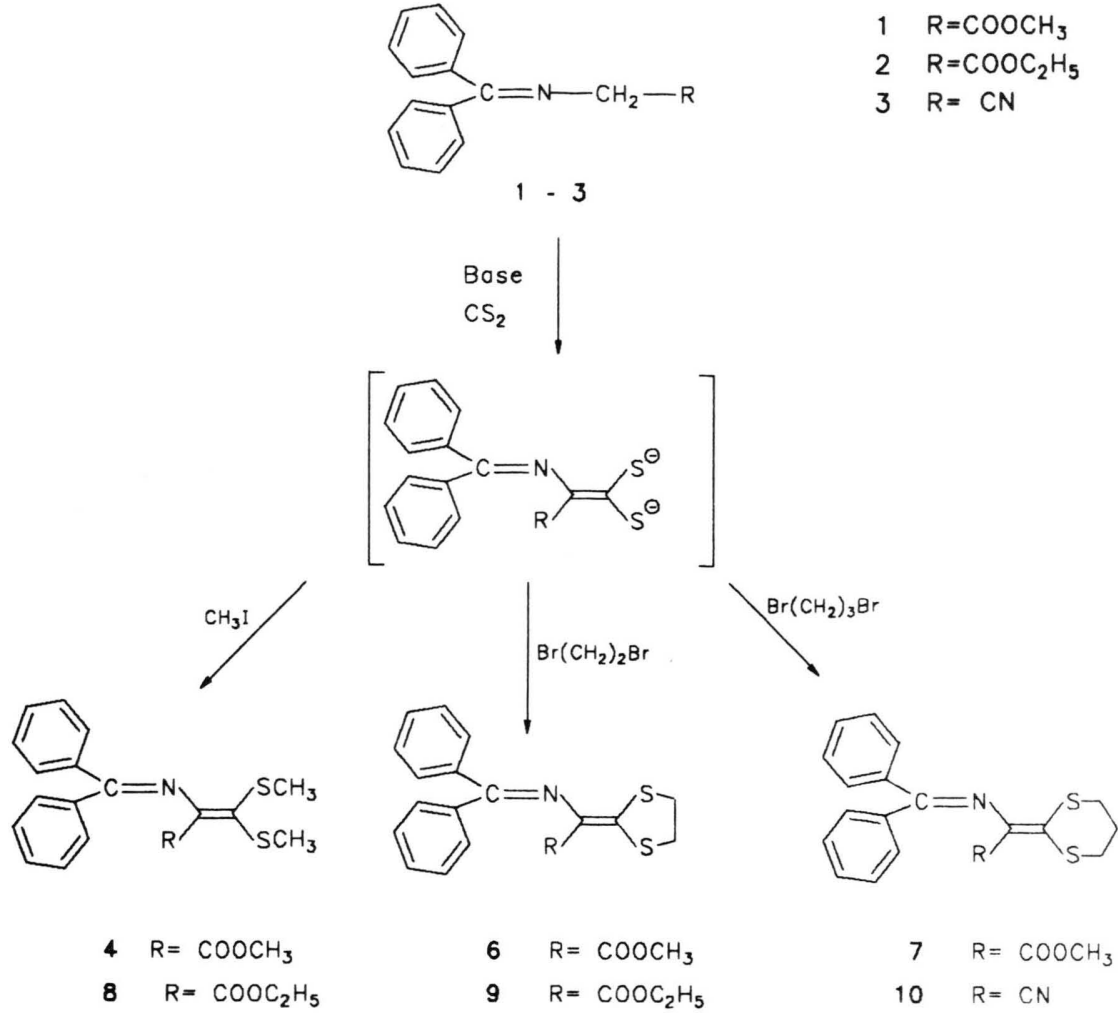

Schema 1 .

$$
\begin{array}{rl}
4 & \mathrm{R}=\mathrm{COOCH}_{3} \\
8 & \mathrm{R}=\mathrm{COOC}_{2} \mathrm{H}_{5} \\
11 & \mathrm{R}=\mathrm{CN}
\end{array}
$$<smiles>[R]C(N=C([S-])c1ccccc1)=C1CCCCC1</smiles>

Dihalogenid, wie 1,2-Dibromethan oder 1,3-Dibrompropan, verwendet.

Die Bildung von öligen, uneinheitlichen Produkten bei der Dithiocarboxylierung und anschlieBender Alkylierung wurde verstärkt bei der Synthese der Verbindungen $\mathbf{4 , 8}$ und 9 beobachtet.

Bei Erhöhung der Akzeptorwirkung am Carbanion und unter Verwendung von $\alpha, \omega$-Dihalogenverbindungen zur Alkylierung isolierten wir kristalline Verbindungen.

Interessante Ergebnisse erhielten wir bei der säulenchromatographischen Aufarbeitung des Reaktionsgemisches, welches bei der Darstellung von 4 anfiel.

Neben 4 isolierten wir in 10\% Ausbeute eine Verbindung, die im ${ }^{1} \mathrm{H}-\mathrm{NMR}$-Spektrum ein Signal für ein vinylisches Proton bei 6,99 ppm zeigt. Das JMODXH- ${ }^{13} \mathrm{C}$-Spektrum zeigt bei $135,71 \mathrm{ppm}$ ein Methin-Kohlenstoffatom. Die in den Spektren der Verbindung 4 erkennbaren Signale für $\mathrm{OCH}_{3}$ bei $3,53 \mathrm{ppm}\left({ }^{1} \mathrm{H}-\mathrm{NMR}\right)$ und $51,82 \mathrm{ppm}$ bzw. für die Carbonylgruppe bei $170,89 \mathrm{ppm}\left({ }^{13} \mathrm{C}-\mathrm{NMR}\right)$, tre- ten bei dieser Verbindung nicht mehr auf. Offensichtlich muß eine Decarboxylierung der Estergruppe stattgefunden haben. Wir schlußfolgern daraus, daß höchstwahrscheinlich neben $\mathbf{4}$ noch 1,1-Bis(methylthio)-2-(diphenylmethylenamino)ethen 5 entstanden ist.<smiles>CSC(=CN=C(c1ccccc1)c1ccccc1)SC</smiles>

Schema 2. 5

Analog dazu finden wir im Spektrum des Reaktionsgemisches von 8 ein Signal bei 6,89 ppm, das ebenfalls einem vinylischen Proton zugeordnet werden könnte.

Die Spektren der Produkte der Dithiocarboxylierungen unter Verwendung von Na- $t$-Butylat als Base zeigen auffälligerweise diese Signale nicht. 
Wie finden für die Ester im IR-Spektrum die Bande für die Carbonyl-Valenzschwingung im Erwartungsbereich um $1700 \mathrm{~cm}^{-1}$ und für die Nitrile die C-N-Absorptionsbande um $2200 \mathrm{~cm}^{-1}$.

In den ${ }^{13} \mathrm{C}$-NMR-Spektren sind charakteristische Verschiebungen für die C-Atome der Phenylringe im Bereich von 126-140 ppm zu finden. Das Signal für die C-N-Doppelbindung ist im Bereich von 163-168 ppm erkennbar. Für die $\mathrm{SCH}_{3}$ Gruppen beobachten wir Signale zwischen 16 und $19 \mathrm{ppm}$.

In Anlehnung an die Dithiocarboxylierungen der Ausgangsverbindungen 1-3 war auch die Thiocarbamoylierung mit Phenylisothiocyanat, einem weiteren Vertreter der Heterokumulene, zu prüfen.

Eine Vielzahl von Arbeiten zur Umsetzung von $\mathrm{CH}$-aciden Verbindungen mit Phenylisothiocyanat sind in der Literatur bekannt [10-13].

Die Produkte 13 und 14, die durch Alkylierung des Intermediates 12 mit Methyliodid bzw. Cyclohexyliodid entstanden, konnten isoliert und identifiziert werden. Bei Verbindung 13 handelt es sich um das 3-Methylthio-3-phenylamino-2-(diphenylmethylenamino)-acrylnitril, welches in $51 \%$ Ausbeute erhalten wurde.
Im IR-Spektrum erscheinen die Aromatenschwingungen bei 3040,3053 sowie $3066 \mathrm{~cm}^{-1}$, die $\mathrm{CH}$-Valenzschwingung für die $\mathrm{SCH}_{3}$-Gruppe bei $2930 \mathrm{~cm}^{-1}$, die NH-Schwingung bei $3209 \mathrm{~cm}^{-1}$ und die Absorptionsbande der Nitrilgruppe bei 2200 $\mathrm{cm}^{-1}$.

Im Protonenspektrum der Verbindung 13 finden wir ein doppeltes Singulett der $\mathrm{SCH}_{3}$-Gruppe bei $2,13 / 2,17 \mathrm{ppm}$ (zurückzuführen auf $E / Z$-Isomerie) und ein Multiplett für die 15 aromatischen Protonen im Bereich von 7,15-7,67 ppm. Das $\mathrm{NH}$-Proton liefert ein verbreitertes Singulett bei $8,02 \mathrm{ppm}$.

Zur Bestätigung des Strukturvorschlages von $\mathbf{1 3}$ wurden außerdem ein ${ }^{1} \mathrm{H}$-rauschentkoppeltes und ein JMODXH- $-{ }^{13} \mathrm{C}$-Spektrum aufgenommen.

Bei der Verwendung von Chloracetylchlorid als Alkylierungsmittel erhielten wir ein $Z / E$-Isomerengemisch von 2-(Diphenylmethylenamino)-2-(4oxo-3-phenyl-1,3-thiazolidin-2-yliden)-acetonitril 15.

Das ${ }^{1} \mathrm{H}-\mathrm{NMR}-$ Spektrum zeigt bei 3,94 ppm und 4,06 ppm zwei Signale für $\mathrm{SCH}_{2}$-Gruppen im Intensitätsverhältnis $80: 20$. Außerdem finden wir im ${ }^{13} \mathrm{C}$-NMR-Spektrum ebenfalls zwei Signale für $\mathrm{SCH}_{2}$-Gruppen bei $30,90 \mathrm{ppm}$ und $32,49 \mathrm{ppm}$.
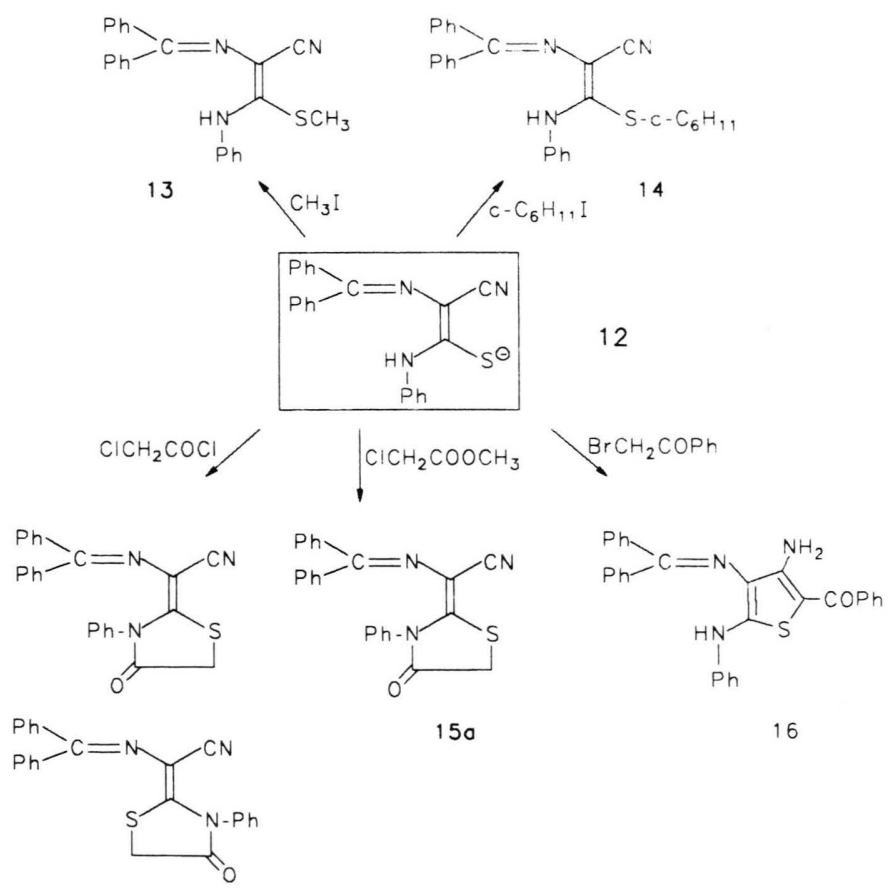

$15 a$

16 


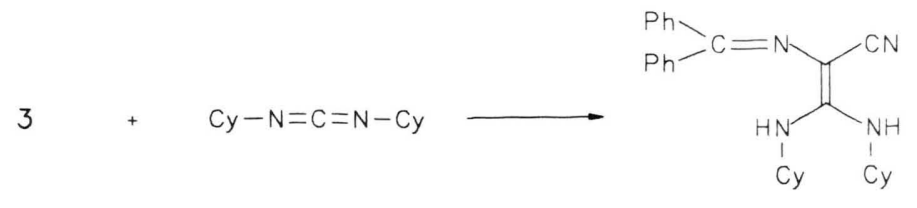

Schema 4.

17

Eine konkrete Zuordnung der einzelnen Signale zum entsprechenden Isomer ist uns derzeitig noch nicht möglich.

Thiazolidone bilden sich, wenn als Alkylierungsmittel Bromessigsäuremethylester verwendet wird [13].

Massen- und schwingungsspektroskopische Daten sowie die Elementaranalyse und Kernresonanzspektren belegen, daß das 2-(Diphenylmethylenamino)-2-(4-oxo-3-phenyl-1,3-thiazolidin-2-yliden)-acetonitril 15 a entstanden ist.

Durch Reaktion von 3 mit Phenylisothiocyanat und Bromacetophenon erhielten wir durch Thorpe-Ziegler-Cyclisierung in $81 \%$ Ausbeute 3Amino-4-(diphenylmethylenamino)-2-benzoyl-5phenylamino-thiophen $\mathbf{1 6}$ in Form orangefarbener Kristalle.
Die Reaktionen des Diphenylmethylenaminoessigsäureethylesters 2 mit Dicyclohexylcarbodiimid ergaben ölige, uneinheitliche Produktgemische mit hohem Dicyclohexylharnstoff-Anteil. Eine saubere analytische Aufarbeitung zur Isolierung der zu erwartenden Zielverbindungen gelang uns nicht.

Setzt man jedoch Verbindung 3 mit DCC bei $-35{ }^{\circ} \mathrm{C}$ um, dann isoliert man nach Umkristallisation aus Ether eine hellgelbe, kristalline Substanz, das 1,1-Bis(cyclohexylamino)-2-(diphenylmethylen-amino)-acrylnitril 17.

So zeigt das IR-Spektrum charakteristische Banden für die CN-Gruppe bei $2160 \mathrm{~cm}^{-1}$, CHund $\mathrm{CH}_{2}$-Valenzschwingungsbanden bei 2855 $\mathrm{cm}^{-1}$ und $2933 \mathrm{~cm}^{-1}$ und $\mathrm{NH}-\mathrm{Banden}$ bei 3300 $\mathrm{cm}^{-1}$ bzw. $3320 \mathrm{~cm}^{-1}$. Bei Aufnahme des IR-Spek-

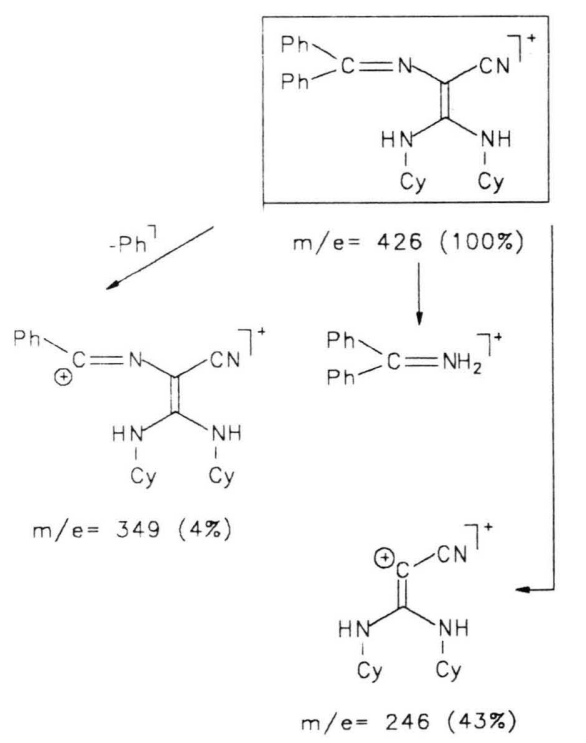

17

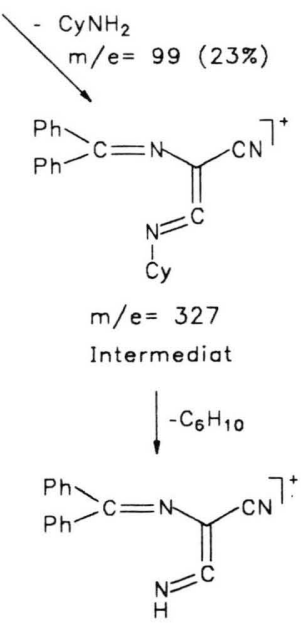

Abb. 1. Massenspektrometrische Fragmentierung von Verbindung 17. 
trums mit einer 2-proz. $\mathrm{CCl}_{4}$-Lösung von 17 treten zusätzliche Banden bei $3373 \mathrm{~cm}^{-1}$ und $3423 \mathrm{~cm}^{-1}$ durch intermolekulare $\mathrm{NH}-$ Wechselwirkungen auf. Die ${ }^{1} \mathrm{H}$-NMR- und ${ }^{13} \mathrm{C}$-NMR-Spektren bestätigen unseren Strukturvorschlag.

Aus den Arbeiten von Dalgaard et al. [14] ist ersichtlich, daß auch Dithiocarboxylierungen $\mathrm{CH}$ acider Systeme unter Phasentransfer-Bedingungen möglich sind.

In früheren Arbeiten haben wir Reaktionen methylenaktiver Verbindungen mit Schwefelkohlenstoff beschrieben. So gelangten wir bei unseren Untersuchungen zur Dithiocarboxylierung $\mathrm{CH}$ acider Thio- und Dithiokohlensäure-Derivate neben 1,3-Dithiolanen bzw. 1,3-Dithianen auch zu neuartigen Keten-S,S-acetalen [15, 16].

Interessant ist, daß unter Phasentransfer-Bedingungen die Synthese von S-(1-Benzoyl-2-bis-methylthio)vinyl-pentamethylen-dithiocarbamaten gelang. Versuche, diese Verbindungen im System $\mathrm{NaH} / \mathrm{THF}$ darzustellen, führten immer zum 1,3Dithiol-2-thion-Derivat [15].

In Anlehnung an die Arbeiten von Augustin und Mitarbeitern [16] dithiocarboxylierten wir die Ausgangsverbindungen 1-3 unter PhasentransferBedingungen im Zweiphasensystem $\mathrm{CHCl}_{3} /$ wäßrige $\mathrm{KOH} /$ Triethylbenzyl-ammoniumchlorid (TEBA), wobei TEBA in äquimolaren Mengen zugegeben wurde.

Die Verbindungen 6, 9, 10 und 11 wurden nach dieser Synthesevariante dargestellt.

Interessant ist, daß bei dieser Verfahrensweise, gegenüber der mit NaH/DMF, deutliche Ausbeuteverbesserungen (im allgemeinen um 100\%) zu verzeichnen waren.

Das IR-Spektrum der Verbindung 11 zeigt eine deutliche Bande bei $2205 \mathrm{~cm}^{-1}$ für die Nitrilgruppe und bei $2915 \mathrm{~cm}^{-1}$ für die $\mathrm{CH}$-Valenzschwingung der Methylgruppen.

Ferner beobachten wir zwei Signale für die $\mathrm{SCH}_{3}$-Gruppen bei 2,32 ppm und 2,46 ppm im ${ }^{1} \mathrm{H}$ NMR-Spektrum.

Bei Verbindung 10 beobachten wir die InfrarotSchwingungsbande für die Nitrilgruppe bei 2200 $\mathrm{cm}^{-1}$.

Im breitbandentkoppelten ${ }^{13} \mathrm{C}$-NMR-Spektrum konnten alle Signale den entsprechenden Kohlenstoffatomen zugeordnet werden. Die von uns dargestellten Verbindungen (vgl. Schema 1) besitzen mehrere Zentren, die Folgereaktionen erwarten lassen. Gompper und Töpf1 [17] haben gezeigt, daß Keten-S,S-acetale mit aromatischen und aliphatischen Aminen und Diaminen zu Keten-S,N-acetalen und Ketenaminalen reagieren können.

Es sollte untersucht werden, inwieweit sich diese Reaktionen auf die von uns synthetisierten Acetale mit Thiomethylgruppen anwenden lassen.

Die Verbindungen 4, 8 und 11 wurden in Ethanol mit $o$-Phenylendiamin bzw. Ethylendiamin $5 \mathrm{~h}$ unter Rückfluß erhitzt. Anschließend wurde die Reaktionslösung auf Eis/Wasser gegossen. Es gelang jedoch in keinem Fall, die entsprechenden Keten-S,N- bzw. N,N-acetale zu isolieren.

Um das dynamische Verhalten der Verbindung 10 beobachten zu können, fertigten wir ${ }^{1} \mathrm{H}$-Tieftemperatur- und ${ }^{13} \mathrm{C}$-Hochtemperatur-Kernresonanzspektren an. Für die $\mathrm{SCH}_{2}$-Gruppen des 1,3Dithian-Systems der Verbindung 10 beobachten wir bei R.T. ${ }^{13} \mathrm{C}$-Verschiebungen bei $28,58 \mathrm{ppm}$ und $29,15 \mathrm{ppm}$, für die $\mathrm{CH}_{2}$-Gruppe bei 23,96 ppm. Durch Temperaturerhöhung, und eine damit eventuell auftretende Rotation um die C-C-Doppelbindung, verbunden mit einer besseren Delokalisation des $\pi$-Systems im Molekül, wäre es möglich, daß beide Signale für die $\mathrm{SCH}_{2}$-Gruppen im Spektrum zusammenfallen. Die chemischen Verschiebungen bleiben jedoch bis zu einer Meßtemperatur von $336 \mathrm{~K}$ unverändert.

Vergleichbare Ergebnisse erhielten wir aus ${ }^{1} \mathrm{H}$ NMR-Tieftemperaturspektren von $\mathbf{1 0}$.

Zur Interpretation der Molekülgeometrie der Verbindungen $\mathbf{4}$ und $\mathbf{1 0}$ wurden SYBYL- und PIMM-Berechnungen [18, 20] durchgeführt, die zeigen, daß die transoide Konformation gegenüber der cisoiden energetisch bevorzugt ist. Ähnliche Ergebnisse fanden Würthwein und Mitarbeiter [19] bei Untersuchungen zur Konformation von 2-Aza-buta-1,3-dienen. Um die geometrischen Gesichtspunkte der Struktur näher zu verstehen, wurde eine Röntgenkristallstrukturanalyse von Verbindung 7 durchgeführt. Im folgenden werden deren Ergebnisse diskutiert.

\section{Molekülstruktur von 7}

Die Molekülstruktur der Verbindung 7 ist in Abb. 2 dargestellt. Relevante Bindungslängen, -winkel und Torsionswinkel können den Tabellen I und II entnommen werden.

Die Verbindung 7 kann als mehrfach substituiertes 2-Aza-1,3-butadien aufgefaßt werden. Ihre 
Tab. I. Ausgewählte Bindungslängen (pm) und Bindungswinkel ()$^{\circ}$ in 7 (Standardabweichungen in Klammern).

\begin{tabular}{|c|c|c|c|}
\hline Atome & Abstand & Atome & Winkel \\
\hline $\mathrm{S} 1-\mathrm{C} 1$ & $175,8(3)$ & $\mathrm{C} 1-\mathrm{S} 1-\mathrm{C} 5$ & $99,5(1)$ \\
\hline $\mathrm{S} 1-\mathrm{C} 5$ & $180,0(4)$ & $\mathrm{C} 1-\mathrm{S} 2-\mathrm{C}_{7}$ & $101,3(2)$ \\
\hline $\mathrm{S} 2-\mathrm{C} 1$ & $175,7(3)$ & $\mathrm{C} 2-\mathrm{N} 1-\mathrm{C} 4$ & $121,6(2)$ \\
\hline $\mathrm{S} 2-\mathrm{C} 7$ & $181,1(4)$ & $\mathrm{S} 1-\mathrm{C} 1-\mathrm{S} 2$ & $118,6(1)$ \\
\hline $\mathrm{O} 1-\mathrm{C}_{3}$ & $120,7(4)$ & $\mathrm{S} 1-\mathrm{C} 1-\mathrm{C} 2$ & $124,2(2)$ \\
\hline $\mathrm{O} 2-\mathrm{C} 3$ & $134,5(3)$ & $\mathrm{S} 2-\mathrm{C} 1-\mathrm{C} 2$ & $117,2(2)$ \\
\hline $\mathrm{O} 2-\mathrm{C} 8$ & $144,0(4)$ & $\mathrm{N} 1-\mathrm{C} 2-\mathrm{C} 1$ & $119,9(2)$ \\
\hline $\mathrm{N} 1-\mathrm{C} 2$ & $142,4(3)$ & $\mathrm{N} 1-\mathrm{C} 2-\mathrm{C} 3$ & $117,3(2)$ \\
\hline $\mathrm{N} 1-\mathrm{C} 4$ & $127,7(3)$ & $\mathrm{C} 1-\mathrm{C} 2-\mathrm{C} 3$ & $122,1(2)$ \\
\hline $\mathrm{C} 1-\mathrm{C} 2$ & $135,0(4)$ & $\mathrm{N} 1-\mathrm{C} 4-\mathrm{C} 9$ & $124,3(2)$ \\
\hline $\mathrm{C} 2-\mathrm{C} 3$ & $147,7(4)$ & $\mathrm{N} 1-\mathrm{C} 4-\mathrm{C} 15$ & $117,5(2)$ \\
\hline $\mathrm{C} 4-\mathrm{C} 9$ & $150,0(4)$ & $\mathrm{C} 9-\mathrm{C} 4-\mathrm{C} 15$ & $118,2(2)$ \\
\hline $\mathrm{C} 4-\mathrm{C} 15$ & $149,0(3)$ & $\mathrm{S} 1-\mathrm{C} 5-\mathrm{C} 6$ & $114,9(2)$ \\
\hline C $5-C 6$ & $150,4(6)$ & $\mathrm{C}^{5}-\mathrm{C}_{6}-\mathrm{C}$ & $114,4(3)$ \\
\hline $\mathrm{C} 6-\mathrm{C}^{7}$ & $150,3(5)$ & $\mathrm{S} 2-\mathrm{C} 7-\mathrm{C} 6$ & $114,5(2)$ \\
\hline
\end{tabular}

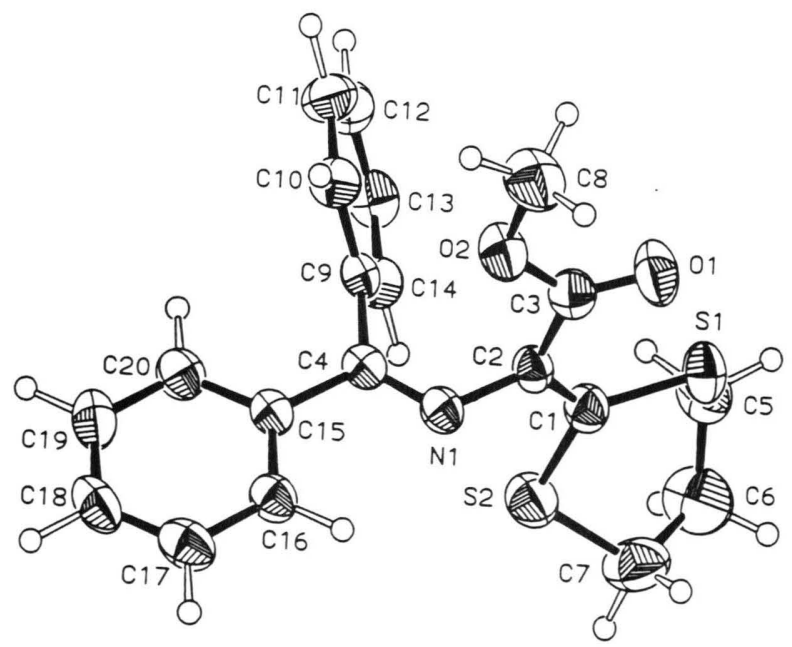

Abb. 2. Molekülstruktur von 7 mit Atomnumerierung (ORTEP II-Darstellung [33], 50\%-Wahrscheinlichkeitsellipsoide, H-Atome in willkürlicher Größe dargestellt).
Molekülstruktur weist, ebenso wie das unsubstituierte 2-Aza-1,3-butadien (a), gut lokalisierte Doppelbindungen auf (vgl. Tab. III). Demgegenüber wird in der ebenfalls mehrfach substituierten Verbindung (E,E)-4-Amino-3-cyan-1-(4-hydroxyphenyl)-4-methoxy-2-aza-1,3-butadien (b) ein geringerer Doppelbindungscharakter und, damit einhergehend, eine Konjugation unter Einbeziehung des Hydroxyphenylsubstituenten gefunden. In 7 verhindert dagegen die durch die beiden Phenylsubstituenten bedingte starke sterische Beanspruchung des Atoms C4 eine derartige Konjugation. Ein analoger Befund wird auch bei der entsprechend substituierten Verbindung 4-Benzoyloxy-1,1,2-triphenyl-azadien (c) registriert. Allerdings ist hier die Azabutadien-Kette bei einem Torsionswinkel $\mathrm{C}=\mathrm{C}-\mathrm{N}=\mathrm{C}$ von $178,3(6)^{\circ}$ planar ausgebildet, während dieser Torsionswinkel in 7 einen Wert von $-103,5(3)^{\circ}$ annimmt.

Ursache hierfür sollte die intramolekulare S 1 ‥ O 1-Wechselwirkung mit einem Abstand von 284,3(2) pm sein, die zudem zu einer weitgehenden Einebnung der Atomgruppierung S1-C 1-C2C3-O1 führt (die größte Abweichung eines Atoms von der durch die fünf genannten Atome definierten Ausgleichsebene beträgt 12,5(3) pm für das Atom C 3).

Während im reinen 1,3-Dithian [23] und in den meisten strukturanalytisch untersuchten 1,3-Dithian-Derivaten die Sesselkonformation im entsprechenden 1,3-Dithianring bevorzugt wird (siehe z. B. [24] bis [27]), liegt in der Verbindung 7 eine gut angenäherte Twist-boat-Konformation vor. Eine Faltungsanalyse nach Cremer und Pople [28] liefert für diesen Ring die folgenden Werte: $\Phi_{2}=$ $-92,0(2)^{\circ}$ und $\theta=91,3(2)^{\circ}$ bei Idealwerten für eine reine Twist-boat-Konformation von $90,0^{\circ}$ und 90, $0^{\circ}$. Dieses Ergebnis steht im Zusammenhang

\begin{tabular}{|c|c|c|c|}
\hline Atome & Winkel & Atome & Winkel \\
\hline $\mathrm{C} 5-\mathrm{S} 1-\mathrm{C} 1-\mathrm{S} 2$ & $30,2(2)$ & $\mathrm{S} 1-\mathrm{C} 1-\mathrm{C}_{2}-\mathrm{N}_{1}$ & $-173,8(2)$ \\
\hline $\mathrm{C} 5-\mathrm{S} 1-\mathrm{C} 1-\mathrm{C} 2$ & $-148,6(2)$ & $\mathrm{S} 1-\mathrm{C} 1-\mathrm{C} 2-\mathrm{C} 3$ & $-\quad 3,2(3)$ \\
\hline $\mathrm{C} 1-\mathrm{S} 1-\mathrm{C} 5-\mathrm{C} 6$ & $-68,0(2)$ & $\mathrm{S} 2-\mathrm{C} 1-\mathrm{C} 2-\mathrm{N} 1$ & $7,4(2)$ \\
\hline $\mathrm{C} 7-\mathrm{S} 2-\mathrm{C} 1-\mathrm{S} 1$ & $23,0(2)$ & $\mathrm{S} 2-\mathrm{C} 1-\mathrm{C} 2-\mathrm{C} 3$ & $178,0(2)$ \\
\hline $\mathrm{C} 7-\mathrm{S} 2-\mathrm{C} 1-\mathrm{C} 2$ & $-158,1(2)$ & $\mathrm{N} 1-\mathrm{C} 4-\mathrm{C} 9-\mathrm{C} 10$ & $-107,8(3)$ \\
\hline $\mathrm{C} 1-\mathrm{S} 2-\mathrm{C} 7-\mathrm{C} 6$ & $-64,1(3)$ & $\mathrm{N} 1-\mathrm{C} 4-\mathrm{C} 9-\mathrm{C} 14$ & $73,9(3)$ \\
\hline $\mathrm{C} 4-\mathrm{N} 1-\mathrm{C}_{2}-\mathrm{C}_{1}$ & $-103,5(3)$ & $\mathrm{N} 1-\mathrm{C} 4-\mathrm{C} 15-\mathrm{C} 16$ & $1,9(3)$ \\
\hline $\mathrm{C} 4-\mathrm{N} 1-\mathrm{C}_{2}-\mathrm{C}_{3}$ & $85,5(3)$ & $\mathrm{N} 1-\mathrm{C} 4-\mathrm{C} 15-\mathrm{C} 20$ & $-177,0(3)$ \\
\hline $\mathrm{C}_{2}-\mathrm{N}_{1}-\mathrm{C}_{4}-\mathrm{C} 9$ & $5,5(3)$ & $\mathrm{S} 1-\mathrm{C} 5-\mathrm{C} 6-\mathrm{C} 7$ & $35,6(3)$ \\
\hline $\mathrm{C} 2-\mathrm{N} 1-\mathrm{C} 4-\mathrm{C} 15$ & $-176,9(2)$ & $\mathrm{C} 5-\mathrm{C} 6-\mathrm{C} 7-\mathrm{S} 2$ & $35,2(3)$ \\
\hline
\end{tabular}

Tab. II. Ausgewählte Torsionswinkel ${ }^{\circ}$ ) in 7 (Standardabweichungen in Klammern). 
Tab. III. Vergleich der Bindungslängen in einigen 2-Aza-1,3-butadienen.

\begin{tabular}{lllll}
\hline Verbindung & $\mathrm{C}=\mathrm{C}$ & $\mathrm{C}-\mathrm{N}$ & $\mathrm{N}=\mathrm{C}$ & Literatur \\
\hline $\mathbf{7}$ & $135,0(4)$ & $142,4(3)$ & $127,7(3) \mathrm{pm}$ & \\
a & $131,9(1)$ & $140,3(5)$ & $125,2(3) \mathrm{pm}$ & {$[21]$} \\
$\mathbf{b}$ & $138,6(2)$ & $139,7(2)$ & $128,0(2) \mathrm{pm}$ & {$[21]$} \\
c & $132,2(8)$ & $140,1(8)$ & $128,1(7) \mathrm{pm}$ & {$[22]$} \\
\hline
\end{tabular}

mit der $\mathrm{sp}^{2}$-Hybridisierung des Atoms $\mathrm{C} 1$, die dazu führt, daß die Gruppierung S 1, S2, C 1, C2 exakt planar ist und damit die Ausbildung der Sesselkonformation verhindert wird. Der Bindungswinkel $\mathrm{S} 1-\mathrm{C} 1-\mathrm{S} 2$ ist mit einem Wert von $118,6(1)^{\circ}$ größer als die gewöhnlich in 1,3-Dithianen beobachteten Werte von etwa 111 bis $115^{\circ}$. Auffällig ist weiterhin eine signifikante Verkürzung der Bindungslängen C $1-\mathrm{S} 1$ (175,8(3) pm) und $\mathrm{C} 1-\mathrm{S} 2(175,7(3) \mathrm{pm})$ im Vergleich zu den üblicherweise beobachteten Werten von etwa 179 bis $182 \mathrm{pm}$ in 1,3-Dithianen. Die beiden in 7 gefundenen Bindungslängen stehen jedoch in sehr guter Übereinstimmung mit dem in der Literatur [15,29] angegebenen Standardwert von 175,1(17) pm für die $\mathrm{C}-\mathrm{S}$-Bindung in einer $\mathrm{C}=\mathrm{C}-\mathrm{S}-\mathrm{C}$-Gruppierung, so daß hier die $\mathrm{C}=\mathrm{C}$-Doppelbindung für die Verkürzung der $\mathrm{C}-\mathrm{S}$-Bindung verantwortlich gemacht werden kann.

Die beiden Phenylringe haben Standardgeometrie. Der Diederwinkel der beiden durch die Phenylringatome gelegten least-squares-Ebenen beträgt $76,3^{\circ}$. Dieser Wert steht mit dem entsprechenden Diederwinkel von $80,8^{\circ}$ in 4-Benzoyloxy1,1,4-triphenyl-azadien [22] in recht guter Übereinstimmung.

\section{Experimenteller Teil}

\section{Allgemeine Bemerkungen}

Die angegebenen Schmelzpunkte wurden auf einem Heiztischmikroskop nach Boetius bestimmt und stellen unkorrigierte Werte dar.

Die Elementaranalysen wurden im automatischen Mikroverfahren mit dem Elementar-Analyser 1104 nach Carlo Erba bzw. im Halbmikromaßstab auf konventionelle Weise angefertigt.

Die Aufnahmen der Infrarot-Spektren erfolgte am Spektralphotometer Specord 75 IR der Firma Carl Zeiss Jena in KBr, Nujol, Chloroform oder Tetrachlorkohlenstoff.
Die ${ }^{1} \mathrm{H}$-NMR- und ${ }^{13} \mathrm{C}-\mathrm{NMR}$-Spektren wurden mit den Impulsspektrometern WP $200(200 \mathrm{MHz})$ und $\mathrm{AC} 80(80 \mathrm{MHz})$ der Firma Bruker in $\mathrm{CDCl}_{3}$ bzw. DMSO- $\mathrm{d}_{6}$ aufgenommen. Die Angaben der chemischen Verschiebung erfolgen in ppm.

Zur Anfertigung von Massenspektren dienten die Geräte MAT-40 des Institutes für Biochemie der Pflanzen Halle und Finnigen MAT-8230 des Institutes für Anorganische Chemie der GeorgAugust-Universität Göttingen.

Die analytische, dünnschichtchromatographische Reinheitskontrolle erfolgte an Silufol-Fertig-

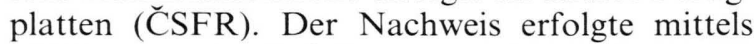
UV-Lampe oder Jod-Kammer. Die Reinigung der Substanzen mittels Säulenchromatographie wurde an Merck-Kieselgel 60 70-230 mesh ASTM durchgeführt. Als mobile Phase für die chromatographische Trennung wurde Ether $/ n$-Hexan 35:65 verwendet.

Destillationen wurden mit der Kugelrohrdestillations-Apparatur GKR-51 (Büchi) durchgeführt. Druck- und Siedepunktsangaben stellen Näherungswerte dar.

Die zur Synthese benötigten Lösungsmittel wurden nach den üblichen Methoden gereinigt.

\section{Allgemeine Arbeitsvorschriften zur Umsetzung von $\mathrm{N}$-Diphenylmethylenglycin-Derivaten mit Heterokumulenen Umsetzungen mit Schwefelkohlenstoff}

\section{Variante A}

$10 \mathrm{mmol}$ der entsprechenden $\mathrm{N}$-geschützten Glycinverbindung 1-2 bzw. des entsprechenden Aminoacetonitrils 3 und $10 \mathrm{mmol}$ Schwefelkohlenstoff werden in $30 \mathrm{ml}$ absolutem Dimethylformamid (DMF) unter Stickstoffatmosphäre bei $-10{ }^{\circ} \mathrm{C}$ portionsweise mit $20 \mathrm{mmol}$ Natriumhydrid ( $80 \%$ in Paraffinöl) versetzt. Nach zweistündigem Rühren bei R.T. versetzt man die Lösung mit $20 \mathrm{mmol}$ eines Alkylierungsmittels $(10 \mathrm{mmol}$ bei Verwendung von 1,2-Dibromethan oder 1,3Dibrompropan). Die Reaktionsmischung wird weitere $2 \mathrm{~h}$ bei R.T. gerührt und dann auf $c a$. $100 \mathrm{~g}$ Eis gegossen. Eventuell ausfallende Kristalle werden abgesaugt und umkristallisiert. Fällt ein öliges Produkt an, wird dieses mit Ether bzw. Chloroform extrahiert, getrocknet und destillativ oder säulenchromatographisch aufgearbeitet.

\section{Variante $B$}

$5 \mathrm{mmol}$ der Verbindung $\mathbf{1 - 3}$ werden in $5 \mathrm{ml}$ Schwefelkohlenstoff und $5 \mathrm{ml}$ Chloroform gelöst und zur doppelt molaren Menge (2,3 g) Triethylbenzyl-ammoniumchlorid (TEBA) und $1,12 \mathrm{~g} \mathrm{(20}$ 
mmol) Kaliumhydroxyd in $10 \mathrm{ml}$ Wasser gegeben. Nachdem 10 min bei R.T. gerührt wurde, tropft man $10 \mathrm{mmol}$ Methyliodid (bzw. $5 \mathrm{mmol} \mathrm{1,2-Di-}$ bromethan oder 1,3-Dibrompropan) zu und läßt $2 \mathrm{~h}$ nachrühren. Die organische Phase wird abgetrennt, zweimal mit Wasser gewaschen, über wasserfreiem Natriumsulfat getrocknet und im Vakuum eingeengt.

\section{3,3-Bis (methylthio)-2-(diphenylmethylenamino)- acrylsäuremethylester $\mathbf{4}$}

nach Variante A, säulenchromatographische Reinigung (2. Fraktion, neben $\mathbf{5}$ ).

Ausbeute: $25,1 \%$ d. Th., rotes Öl.

$\mathrm{C}_{19} \mathrm{H}_{19} \mathrm{NO}_{2} \mathrm{~S}_{2}(357,48)$

EA Ber. C 63,84 H 5,36 N 3,91 S 17,94\%, Gef. C 63,76 H 5,71 N 3,94 S 17,60\%.

IR $\left(\mathrm{CHCl}_{3}\right) v: 1720(\mathrm{C}=\mathrm{O}) \mathrm{cm}^{-1} .{ }^{1} \mathrm{H}-\mathrm{NMR}$ $\left(200 \mathrm{MHz}, \mathrm{CDCl}_{3}\right) \delta: 2,17\left(\mathrm{~s}, 3 \mathrm{H}, \mathrm{SCH}_{3}\right), 2,23(\mathrm{~s}$, $\left.3 \mathrm{H}, \mathrm{SCH}_{3}\right), 3,53\left(\mathrm{~s}, 3 \mathrm{H}, \mathrm{OCH}_{3}\right), 7,27-7,74(\mathrm{~m}$, $10 \mathrm{H}$, aromat.). ${ }^{13} \mathrm{C}-\mathrm{NMR}\left(200 \mathrm{MHz}, \mathrm{CDCl}_{3}\right)$ $\delta: \quad 16,07\left(\mathrm{SCH}_{3}\right), 18,69\left(\mathrm{SCH}_{3}\right), 51,82\left(\mathrm{OCH}_{3}\right)$, $108,27(\mathrm{C}=\mathrm{C}), 127,85,128,12 ; 128,45 ; 128,56$; 128,$73 ; \quad 128,98 ; \quad$ (C-aromat.); 129,12; 129,64; 130,$12 ; 131,09 ; 136,68 ; 138,56 ; 143,09 \quad(\mathrm{C}=\mathrm{C})$; $164,12(\mathrm{C}=\mathrm{N}), 170,89(\mathrm{C}=\mathrm{O})$.

\section{1,1-Bis (methylthio)-2-(diphenylmethylenamino)- ethen 5}

nach Variante A, säulenchromatographische Reinigung (1. Fraktion, neben 4 ).

Ausbeute: $10 \%$ d. Th., rotes Öl.

$\mathrm{C}_{17} \mathrm{H}_{17} \mathrm{NS}_{2}(299,44)$

EA Ber. C 68,18 H 5,72 N 4,67 S 21,41\%, Gef. C 67,98 H 5,63 N 4,60 S 21,01\%.

${ }^{1} \mathrm{H}-\mathrm{NMR}\left(200 \mathrm{MHz}, \mathrm{CDCl}_{3}\right) \delta: 2,22(\mathrm{~s}, 3 \mathrm{H}$, $\left.\mathrm{SCH}_{3}\right), 2,51\left(\mathrm{~s}, 3 \mathrm{H}, \mathrm{SCH}_{3}\right), 6,99(\mathrm{~s}, 1 \mathrm{H}, \mathrm{CH})$, 7,17-7,75 (m, $10 \mathrm{H}$, aromat.). ${ }^{13} \mathrm{C}-\mathrm{NMR}(200$ $\left.\mathrm{MHz}, \mathrm{CDCl}_{3}\right) \delta: 16,20\left(\mathrm{SCH}_{3}\right), 17,57\left(\mathrm{SCH}_{3}\right)$, 128,$03 ; \quad 128,44 ; \quad 128,57 ; \quad 128,70 ; \quad$ (C-aromat.), 128,$77 ; \quad 130,13 ; 135,89 ; 139,32 ; 135,71 \quad(\mathrm{CH})$, $163,62(\mathrm{C}=\mathrm{N})$.

2-( Diphenylmethylenamino)-2-(1,3-dithiolan-

2-yliden)-essigsäuremethylester 6

Nach Variante A oder B.

Ausbeute: $28 \%$ d. Th., rote Kristalle, F.p.: $109-$ $112^{\circ} \mathrm{C}($-Butanol).

$\mathrm{C}_{19} \mathrm{H}_{17} \mathrm{NO}_{2} \mathrm{~S}_{2}(355,46)$

EA Ber. C 64,20 H 4,82 N 3,94 S 18,04\%, Gef. C 64,15 H 4,80 N 3,90 S 18,01\%.
${ }^{1} \mathrm{H}-\mathrm{NMR}\left(200 \mathrm{MHz}, \mathrm{CDCl}_{3}\right) \delta: 3,26-3,47(\mathrm{~m}$, $\left.4 \mathrm{H}, 2 \times \mathrm{SCH}_{2}\right), 3,40\left(\mathrm{~s}, 3 \mathrm{H}, \mathrm{OCH}_{3}\right), 7,15-7,76(\mathrm{~m}$, $10 \mathrm{H}$, aromat.).

\section{2-(Diphenylmethylenamino)-2-( 1,3-dithian- \\ 2-yliden)-essigsäuremethylester 7}

Nach Variante A.

Ausbeute: $22 \%$ d. Th., gelbe Kristalle, F.p.: $117-120{ }^{\circ} \mathrm{C}$ ( $n$-Butanol).

$\mathrm{C}_{20} \mathrm{H}_{19} \mathrm{NO}_{2} \mathrm{~S}_{2}(369,51)$

EA Ber. C 65,01 H 5,18 N 3,79 S 17,36\%, Gef. C 64,99 H 5,20 N 3,37 S 17,93\%.

${ }^{1} \mathrm{H}-\mathrm{NMR}\left(200 \mathrm{MHz}, \mathrm{CDCl}_{3}\right) \delta: 2,05-2,18(\mathrm{~m}$, $\left.2 \mathrm{H}, \mathrm{CH}_{2}\right), 2,83-2,95\left(\mathrm{~m}, 4 \mathrm{H}, 2 \times \mathrm{SCH}_{2}\right), 3,45(\mathrm{~s}$, $\left.3 \mathrm{H}, \mathrm{OCH}_{3}\right), 7,14-7,75$ (m, $10 \mathrm{H}$, aromat.).

\section{1,1-Bis (methylthio)-2-(diphenylmethylenamino)- acrylsäureethylester $\mathbf{8}$}

Nach Variante A.

Ausbeute: $21,6 \%$ d. Th., rotes Öl, K.p. $185^{\circ} \mathrm{C}$.

$\mathrm{C}_{20} \mathrm{H}_{21} \mathrm{NO}_{2} \mathrm{~S}_{2}(371,51)$

EA Ber. C 64,66 H 5,70 N 3,77 S 17,26\%, Gef. C 64,56 H 5,75 N 3,72 S 17,20\%.

${ }^{1} \mathrm{H}-\mathrm{NMR}\left(80 \mathrm{MHz}, \mathrm{CDCl}_{3}\right) \delta: 1,24(\mathrm{t}, 3 \mathrm{H}$, $\left.\mathrm{CH}_{3}\right), 3,98$ (q, $\left.2 \mathrm{H}, \mathrm{CH}_{2}\right), 2,19\left(\mathrm{~s}, 3 \mathrm{H}, \mathrm{SCH}_{3}\right), 2,48$ (s, $3 \mathrm{H}, \mathrm{SCH}_{3}$ ), 7,13-7,83 (m, $10 \mathrm{H}$, aromat.). - IR (kap.) v: $1715(\mathrm{C}=\mathrm{O}) ; 2853 ; 2918 ; 2949 ; 2980$ $\left(\mathrm{CH}_{2}-, \mathrm{CH}_{3}\right.$-Valenz) $3017 ; 3053 ; 3078$ (Aromaten) $\mathrm{cm}^{-1}$.

\section{2-(Diphenylmethylenamino)-2-(1,3-dithiolan- 2-yliden)-essigsäureethylester 9}

Nach Variante A oder B.

Ausbeute: $27,1-49,0 \%$ d. Th., rotes Öl, K.p. $150{ }^{\circ} \mathrm{C}$.

$\mathrm{C}_{20} \mathrm{H}_{19} \mathrm{NO}_{2} \mathrm{~S}_{2}(369,49)$

EA Ber. C 65,01 H 5,18 N 3,79 S 17,36\%, Gef. C 64,83 H 5,11 N 3,69 S 17,35\%.

IR $\left(\mathrm{CCl}_{4}\right) v: 1740(\mathrm{C}=\mathrm{O}) \mathrm{cm}^{-1} .{ }^{1} \mathrm{H}-\mathrm{NMR}(200$ $\mathrm{MHz}, \mathrm{CDCl}_{3}$ ) $\delta: 1,03 / 1,27$ (t, $3 \mathrm{H}, \mathrm{CH}_{3}$ ), $1: 4$ Isomerengemisch, $3,28-3,63\left(\mathrm{~m}, 4 \mathrm{H}, 2 \times \mathrm{SCH}_{2}\right), 3,85$ $4,23\left(\mathrm{q}, 2 \mathrm{H}, \mathrm{CH}_{2}\right), 1: 4$ Isomerengemisch, 7,187,79 (m, $10 \mathrm{H}$, aromat.). $-{ }^{13} \mathrm{C}-\mathrm{NMR}(80 \mathrm{MHz}$, $\left.\mathrm{CDCl}_{3}\right) \quad \delta: \quad 14,23 \quad\left(\mathrm{CH}_{3}\right), 34,82\left(\mathrm{SCH}_{2}\right), 37,85$ $\left(\mathrm{SCH}_{2}\right), 43,91\left(\mathrm{OCH}_{2}\right), 118,07 \quad(\mathrm{C}=\mathrm{C}), 126,85$; 127,$99 ; 128,14 ; 128,36 ; 129,25 ; 129,54$; (C-aromat.), 130,$05 ; 130,89 ; 132,50 ; 137,39 ; 137,65$; 139,$20 ; \quad 147,05 \quad(\mathrm{C}=\mathrm{C}), \quad 168,50 \quad(\mathrm{C}=\mathrm{N}), \quad 169,70$ $(\mathrm{C}=\mathrm{O})$. 


\section{1,1-Bis(methylthio)-2-(diphenylmethylenamino )- acrylnitril 11}

Nach Variante B.

Ausbeute: $57 \%$ d. Th., rotbraunes Öl.

$\mathrm{C}_{18} \mathrm{H}_{16} \mathrm{~N}_{2} \mathrm{~S}_{2}(324,45)$

EA Ber. C 66,63 H 4,79 N 8,63 S 19,76\%; Gef. C 66,69 H 4,98 N 8,21 S 19,50\%.

IR (kap.) v: $2205(\mathrm{C} \equiv \mathrm{N}) .2915$ (CH-Valenz, $\mathrm{SCH}_{3}$ ), 3015; 3053; 3080 (Aromaten) $\mathrm{cm}^{-1}$. $-{ }^{1} \mathrm{H}-$ NMR $\left(80 \mathrm{MHz}, \mathrm{CDCl}_{3}\right) \delta: 2,32\left(\mathrm{~s}, 3 \mathrm{H}, \mathrm{SCH}_{3}\right)$, $2,46\left(\mathrm{~s}, 3 \mathrm{H}, \mathrm{SCH}_{3}\right), 7,17-7,80(\mathrm{~m}, 10 \mathrm{H}$, aromat.).

\section{2-(Diphenylmethylenamino)-2-(1,3-dithian-}

\section{2-yliden)-acetonitril $\mathbf{1 0}$}

Nach Variante B.

Ausbeute: $40 \%$ d. Th., orangefarbene Kristalle, F.p.: $130-131{ }^{\circ} \mathrm{C}$ (Ethanol/Wasser).

$\mathrm{C}_{19} \mathrm{H}_{16} \mathrm{~N}_{2} \mathrm{~S}_{2}(336,46)$

EA Ber. C 67,82 H 4,79 N 8,32 S 19,05\%, Gef. C 67,11 H 4,54 N 7,99 S 18,18\%.

MS $\left(m / e, \mathrm{M}^{+}\right) 336(100 \%)$. - IR $\left(\mathrm{CHCl}_{3}\right) v$ : $2200(\mathrm{C} \equiv \mathrm{N}), 2927\left(\mathrm{CH}-\right.$ Valenz, $\left.\mathrm{SCH}_{2}\right) \mathrm{cm}^{-1}$. ${ }^{1} \mathrm{H}-\mathrm{NMR}\left(200 \mathrm{MHz}, \mathrm{CDCl}_{3}\right) \delta: 2,25(\mathrm{~m}, 2 \mathrm{H}$, $\left.\mathrm{CH}_{2}\right), 3,04\left(\mathrm{~m}, 4 \mathrm{H}, 2 \times \mathrm{SCH}_{2}\right), 7,34-7,72(\mathrm{~m}, 10 \mathrm{H}$, aromat.). $-{ }^{13} \mathrm{C}-\mathrm{NMR}\left(200 \mathrm{MHz}, \mathrm{CDCl}_{3}\right) \delta: 23,96$ $\left(\mathrm{CH}_{2}\right), \quad 28,58 \quad\left(\mathrm{SCH}_{2}\right), \quad 29,15 \quad\left(\mathrm{SCH}_{2}\right), \quad 113,00$ $(\mathrm{C} \equiv \mathrm{N})^{*}, 114,37(\mathrm{C}=\mathrm{C})^{*}, 128,22 ; 128,47 ; 128,89$; 129,36; (C-aromat.), 129,99; 130,94; 135,88; 139,$00 ; 153,55(\mathrm{C}=\mathrm{C}), 166,11(\mathrm{C}=\mathrm{C})\left({ }^{*}=\right.$ können vertauscht sein).

\section{Umsetzungen mit Phenylisothiocyanat}

\section{Variante $A$}

$10 \mathrm{mmol}$ der entsprechenden $\mathrm{N}$-geschützten Glycinverbindung 1-2 bzw. des entsprechenden Aminoacetonitrils $\mathbf{3}$ und $\mathbf{1 0} \mathrm{mmol}$ Phenylisothiocyanat werden in $30 \mathrm{ml}$ abs. DMF unter Stickstoffatmosphäre bei $-10{ }^{\circ} \mathrm{C}$ durch Zugabe von 10 mmol $\mathrm{NaH}$ zur Reaktion gebracht. Nach zweistündigem Rühren bei R.T. versetzt man die Lösung mit $10 \mathrm{mmol}$ eines Alkylierungsmittels, und rührt weitere $2 \mathrm{~h}$. Anschließend wird das Reaktionsgemisch auf $c a .100 \mathrm{~g}$ Eis gegossen. Eventuell ausfallende Festprodukte werden abgesaugt und umkristallisiert. Ölige Produkte werden mit Ether oder Chloroform extrahiert, getrocknet und destillativ bzw. säulenchromatographisch aufgearbeitet.

\section{Variante B}

Durchführung der Versuche nach Variante A, jedoch Alkylierung mit substituierten $\alpha$-Halogen- ketonen oder Chloracetonitril. Anschließend wird $2 \mathrm{~h}$ bei R.T. gerührt und danach das Reaktionsgemisch auf $c a$. $100 \mathrm{~g}$ Eis gegossen. $5 \mathrm{~g} \mathrm{NaOH}$, in 20 $\mathrm{ml}$ Wasser gelöst, werden dazugegeben $(\mathrm{pH} c a$. $9-10$ ) und weitere $2 \mathrm{~h}$ gerührt. Der ausfallende Feststoff wird abgesaugt, getrocknet und umkristallisiert.

3-Methylthio-3-phenylamino-2-(diphenylmethylenamino)-acrylnitril $\mathbf{1 3}$

Nach Variante A.

Ausbeute: $51,4 \%$ d. Th., gelbe Kristalle, F.p.: 117- $120{ }^{\circ} \mathrm{C}$ (Aceton/Wasser).

$\mathrm{C}_{23} \mathrm{H}_{19} \mathrm{~N}_{3} \mathrm{~S}(369,48)$

EA Ber. C 74,77 H 5,18 N 11,37 S 8,68\%, Gef. C 74,25 H 5,22 N 11,26 S 8,75\%.

IR $(\mathrm{KBr}) v: 2200(\mathrm{C} \equiv \mathrm{N}), 2930\left(\mathrm{SCH}_{3}\right), 3040$; 3053; 3066 (Aromaten), $3209(\mathrm{NH}) \mathrm{cm}^{-1}$. $-{ }^{1} \mathrm{H}-$ NMR $\left(80 \mathrm{MHz}, \mathrm{CDCl}_{3}\right) \delta: 2,13 / 2,17(\mathrm{~s}, 3 \mathrm{H}$, $\mathrm{SCH}_{3}$ ), zwei Isomere, 7,15-7,67 (m, $15 \mathrm{H}$, aromat.), 8,02 (s, $1 \mathrm{H}, \mathrm{NH}) .-{ }^{13} \mathrm{C}-\mathrm{NMR}(200 \mathrm{MHz}$, $\left.\mathrm{CDCl}_{3}\right) \delta: 16,32\left(\mathrm{SCH}_{3}\right), 102,06(\mathrm{C}=\mathrm{C}), 115,17$ $(\mathrm{C} \equiv \mathrm{N}), 122,58 ; 124,66 ; 128,05 ; 128,23 ; 128,48$; 128,76; (C-aromat.), 129,$11 ; 129,65 ; 130,20$; 135,$97 ; 139,53 ; 139,80 ; 152,50 \quad(\mathrm{C}=\mathrm{C}), \quad 161,63$ $(\mathrm{C}=\mathrm{N})$.

\section{3-Cyclohexylthio-3-phenylamino-2-(diphenyl-}

methylenamino)-acrylnitril $\mathbf{1 4}$

Nach Variante A.

Ausbeute: $10 \%$ d. Th., rotes Öl.

$\mathrm{C}_{28} \mathrm{H}_{27} \mathrm{~N}_{3} \mathrm{~S}(437,60)$

EA Ber. C 76,85 H 6,22 N 9,60 S 7,33\%, Gef. C 75,90 H 6,01 N 9,15 S 6,98\%.

IR (kap.) v: $2050(\mathrm{C} \equiv \mathrm{N}), 2856 ; 2925\left(\mathrm{CH}-, \mathrm{CH}_{2}-\right.$ Valenzen), $3290(\mathrm{NH}) \mathrm{cm}^{-1}$.

E/Z-Isomerengemisch von 2-(Diphenylmethylenamino)-2-(4-oxo-3-phenyl-1,3-thiazolidin-

2-yliden)-acetonitril $\mathbf{1 5}$

Nach Variante A.

Ausbeute: $53,6 \%$ d. Th., beigefarbene Kristalle, F.p.: $181-185^{\circ} \mathrm{C}$.

$\mathrm{C}_{24} \mathrm{H}_{17} \mathrm{~N}_{3} \mathrm{OS}(395,47)$

EA Ber. C 72,88 H 4,33 N 10,62 S 8,10\%, Gef. C 72,41 H 4,37 N 10,52 S 8,46\%.

IR $(\mathrm{KBr}) v: 1720(\mathrm{C}=\mathrm{O}), 2190(\mathrm{C} \equiv \mathrm{N}) \mathrm{cm}^{-1}$. ${ }^{1} \mathrm{H}-\mathrm{NMR}\left(80 \mathrm{MHz}, \mathrm{CDCl}_{3}\right) \delta: 3,94 / 4,0620: 80(\mathrm{~s}$, $\left.2 \mathrm{H}, \mathrm{CH}_{2}\right),(2$ Isomere), $6,73-7,62(\mathrm{~m}, 15 \mathrm{H}$, aromat.). $-{ }^{13} \mathrm{C}-\mathrm{NMR}\left(80 \mathrm{MHz}, \mathrm{CDCl}_{3}\right) \delta: 30,90$ $32,94\left(\mathrm{CH}_{2}\right)$ (2 Isomere), 113,50 $(\mathrm{C} \equiv \mathrm{N}), 127,33-$ $138,37(\mathrm{C}$-aromat. $), 172,46(\mathrm{C}=\mathrm{N}), 206,75(\mathrm{C}=\mathrm{O})$. 
2-(Diphenylmethylenamino)-2-(4-oxo-3-phenyl-

1,3-thiazolidin-2-yliden)-acetonitril 15a

Nach Variante B. Alkyliert mit Bromessigsäuremethylester.

Ausbeute: $15,2 \%$ d. Th., beigefarbene Kristalle, F.p.: $225-230{ }^{\circ} \mathrm{C}$ (Aceton/Wasser).

$\mathrm{C}_{24} \mathrm{H}_{17} \mathrm{~N}_{3} \mathrm{OS}(395,47)$

EA Ber. C 72,88 H 4,33 N 10,62 S 8,10\%, Gef. C 72,42 H 4,64 N 9,69 S 8,27\%.

$\operatorname{MS}\left(m / e, \mathrm{M}^{+}\right) 395(100 \%)$, IR (KBr) $v: 1720$ $(\mathrm{C}=\mathrm{O}), 2193(\mathrm{C} \equiv \mathrm{N}) \mathrm{cm}^{-1} .-{ }^{1} \mathrm{H}-\mathrm{NMR}(80 \mathrm{MHz}$, $\left.\mathrm{CDCl}_{3}\right) \delta: 3,93\left(\mathrm{~s}, 2 \mathrm{H}, \mathrm{CH}_{2}\right), 7,15-7,74(\mathrm{~m}, 15 \mathrm{H}$, aromat.).

\section{3-Amino-4-(diphenylmethylenamino)-2-benzoyl- 5-phenylamino-thiophen $\mathbf{1 6}$}

Nach Variante B, alkyliert mit Bromacetophenon.
Ausbeute: $80,7 \%$ d. Th., orangefarbene Kristalle, F.p.: 83-84 C (Methanol/Wasser).

EA Ber. C 76,08 H 4,89 N 2,95 S 6,77\%, Gef. C 75,80 H 4,51 N 3,01 S 6,57\%.

IR $\left(\mathrm{CCl}_{4}\right) v: 1750(\mathrm{C}=\mathrm{O}), 3440\left(\mathrm{NH}, \mathrm{NH}_{2}\right) \mathrm{cm}^{-1}$. - ${ }^{1} \mathrm{H}-\mathrm{NMR}\left(80 \mathrm{MHz}, \mathrm{CDCl}_{3}\right) \delta: 6,83-7,84(\mathrm{~m}$, $20 \mathrm{H}$, aromat.). $-{ }^{13} \mathrm{C}-\mathrm{NMR}\left(200 \mathrm{MHz}, \mathrm{CDCl}_{3}\right) \delta$ : 97,76 (C-Atom 4)*, 123,16 (C-Atom 2)*, 121,38; 122,49; (C-aromat.), 127,11-131,07 (C-aromat.), 138,$80 ; 139,10(4 \times$ quart. C-aromat. und C-Atom 3), 139,99; 140,75;121,23; 151,26 (C-Atom 5), $169,04(\mathrm{C}=\mathrm{N}), 186,59(\mathrm{C}=\mathrm{O}),(*=$ können vertauscht sein).

\section{Umsetzungen mit Dicyclohexylcarboditimid}

$\mathrm{Zu}$ einer Lösung von 2,20 g (10 mmol) Diphenylmethylenaminoacetonitril $\mathbf{3}$ in absolutem DMF gibt man unter Stickstoffatmosphäre 2,06 g (10 mmol) Dicyclohexylcarbodiimid.

\begin{tabular}{|c|c|}
\hline Summenformel & $\mathrm{C}_{20} \mathrm{H}_{19} \mathrm{NO}_{2} \mathrm{~S}_{2}$ \\
\hline Molmasse & $369,51 \mathrm{~g} / \mathrm{mol}$ \\
\hline Gitterkonstanten & $\begin{array}{ll}a=1352,1(2) \mathrm{pm}, & \alpha=90,0^{\circ} \\
b=824,5(1) \mathrm{pm}, & \beta=94,54(1)^{\circ}\end{array}$ \\
\hline Zellvolumen & $\begin{array}{l}c=1660,0(2) \mathrm{pm}, \gamma=90,0^{\circ} \\
\mathrm{V}=1844,7 \cdot 10^{6} \mathrm{pm}^{3}\end{array}$ \\
\hline Formeleinheiten pro Zelle & $Z=4$ \\
\hline $\mathrm{F}(000)$ & 776 \\
\hline Dichte & $\varrho_{\text {röntg }}=1,330 \mathrm{~g} / \mathrm{cm}^{3}$ \\
\hline Kristallsystem, Raumgruppe & monoklin, $\mathrm{P}_{2} / c$ \\
\hline Meßgerät & Vierkreisdiffraktometer Stoe STADI 4 \\
\hline Strahlung, Wellenlänge & $\operatorname{MoK}_{\alpha}$ (Graphitmonochromator), 71,073 pm \\
\hline Kristallgröße & $0,4 \times 0,35 \times 0,25 \mathrm{~mm}$ \\
\hline Meßtemperatur & $293 \mathrm{~K}$ \\
\hline Linearer Absorptionskoeffizient & $\mu=0,29 \mathrm{~mm}^{-1}$ \\
\hline Meßbereich & $2 \theta \leqslant 45,0^{\circ}$ \\
\hline Bereich von $h, k, l$ & $-14 / 14,0 / 8,0 / 17$ und $-14 / 14,-8 / 0,-17 / 0$ \\
\hline $\begin{array}{l}\text { Standardreflexe (max. } \\
\text { Intensitätsschwankung) }\end{array}$ & $231( \pm 3,3 \%), 020( \pm 4,0 \%), 300( \pm 1,9 \%)$ \\
\hline $\begin{array}{l}\text { Zahl der gemessenen } \\
\text { unabhängigen Reflexe }\end{array}$ & 2355 \\
\hline Zahl der beobachteten Reflexe & 2355 , alle Reflexe als beobachtet eingestuft \\
\hline Korrekturen & $\begin{array}{l}\text { Lorentz- und Polarisationskorrektur, } \\
\text { empirische Extinktionskorrektur nach }\end{array}$ \\
\hline Strukturlösung & $\begin{array}{l}\mathrm{F}_{\mathrm{korr}}=\mathrm{F}_{\mathrm{c}}\left(1-10^{-6} \chi \mathrm{F}_{c}{ }^{2} / \sin \theta\right) \text { mit } \chi=0,84(7) \\
\text { direkte Methoden }\end{array}$ \\
\hline Strukturverfeinerung & $\begin{array}{l}\text { Full-Matrix Least-Squares, alle } \\
\text { Nicht-H-Atome mit anisotropen }\end{array}$ \\
\hline & Auslenkungsparametern \\
\hline H-Atomlagen & berechnet \\
\hline Anzahl der verfeinerten Parameter & 239 \\
\hline $\begin{array}{l}\max . \Delta / \sigma \text { im letzten } \\
\text { Verfeinerungszyklus }\end{array}$ & 0,340 \\
\hline Wichtungsschema & $w=0,231 / \sigma^{2}(\mathrm{~F})$ \\
\hline Gütefaktoren & $R=0,044, w R=0,035, \mathrm{~S}=2,02$ \\
\hline $\begin{array}{l}\text { Restelektronendichten in } \\
\text { abschließender } \Delta \mathrm{F} \text {-Map }\end{array}$ & $+0,219$ bzw. $-0,260 \cdot 10^{-6} \mathrm{e} / \mathrm{pm}^{3}$ \\
\hline
\end{tabular}

Tab. IV. Kristalldaten und Angaben zur Röntgenkristallstrukturanalyse von 7 . 
Man kühlt auf $-35^{\circ} \mathrm{C}$, fügt portionsweise 10 mmol $\mathrm{NaH}$ zu, erwärmt das Reaktionsgemisch langsam auf R.T. und läßt $24 \mathrm{~h}$ rühren.

Danach gießt man das Reaktionsgemisch auf $c a$. $100 \mathrm{~g}$ Eis, saugt den Feststoff ab und kristallisiert aus Ether um.

\section{1,1-Bis (cyclohexylamino)-2-(diphenylmethylen- amino)-acrylnitril 17}

Ausbeute: $77,6 \%$ d. Th., hellgelbe Kristalle, F.p.: $172-174{ }^{\circ} \mathrm{C}$ (Ether).

$\mathrm{C}_{28} \mathrm{H}_{34} \mathrm{~N}_{4}(426,60)$

EA Ber. C 78,83 H 8,03 N 13,13\%,

Gef. C 78,05 H 8,17 N 12,56\%.

MS $\left(m / e, \mathrm{M}^{+}\right) 426(100 \%)$. - IR $\left(\mathrm{CCl}_{4}\right) v$ : 1590-1595 ( $\mathrm{C}=\mathrm{N}-\mathrm{C}=\mathrm{C}$ kon. $), 2160 \quad(\mathrm{C} \equiv \mathrm{N})$, 2855; 2933 (CH-, $\mathrm{CH}_{2}$-Valenz), 3300; $3320(\mathrm{NH})$ $\mathrm{cm}^{-1}$. - ${ }^{1} \mathrm{H}-\mathrm{NMR}\left(200 \mathrm{MHZ}, \mathrm{CDCl}_{3}\right) \delta: 1,00-$ $1,96\left(\mathrm{~m}, 20 \mathrm{H}, 10 \times \mathrm{CH}_{2}\right.$-cyclohexyl), 4,23; 6,23 (ds, $2 \mathrm{H}, 2 \times \mathrm{CH}-$ cyclohexyl), 3,31 (s, $2 \mathrm{H}, \mathrm{NH}), 7,38-$ $7,76\left(\mathrm{~m}, 10 \mathrm{H}\right.$, aromat.). $-{ }^{13} \mathrm{C}-\mathrm{NMR}(80 \mathrm{MHz}$, $\left.\mathrm{CDCl}_{3}\right) \delta: 24,75 ; 24,97\left(\mathrm{CH}_{2}\right.$-cyclohexyl $), 25,44$; 53,87; 54,76 (CH-cyclohexyl), 127,04; 128,01 (Caromat.), 129,57.

\section{Röntgenkristallstrukturanalyse von 7}

Geeignete Einkristalle wurden durch Umkristallisation aus $n$-Butanol erhalten. Die Gitterkonstanten wurden über eine Ausgleichsrechnung aus $2 \theta$-Winkeln von 82 Reflexen $\left(20^{\circ}<2 \theta<26^{\circ}\right)$ bestimmt. Die Kristalldaten und Einzelheiten zur Strukturbestimmung sind Tab. IV zu entnehmen. Die ermittelten Atomparameter sind in Tab. V zusammengestellt*. Für die Berechnung wurden die Programme SHELXS 86 [30], SHELX 76 [31] und EDIT [32] benutzt.

Die Autoren sind dem Fonds der Chemischen Industrie für großzügige Unterstützung zu Dank verpflichtet.
Tab. V. Atomkoordinaten und äquivalente isotrope Auslenkungsparameter (in $10^{4} \mathrm{pm}^{2}$ ) für die Nichtwasserstoffatome von 7 (Standardabweichungen in Klammern).

\begin{tabular}{llrll}
\hline Atom & $x / a$ & \multicolumn{1}{l}{$y / b$} & \multicolumn{1}{l}{$z / c$} & \multicolumn{1}{l}{$\mathrm{U}_{\mathrm{eq}}^{*}$} \\
\hline S 1 & $0,48279(2)$ & $0,0348(1)$ & $0,12031(5)$ & $0,0553(3)$ \\
S 2 & $0,59891(6)$ & $0,3480(1)$ & $0,13649(5)$ & $0,0594(3)$ \\
O 1 & $0,6282(2)$ & $-0,2133(3)$ & $0,1445(1)$ & $0,0662(9)$ \\
O2 & $0,7880(1)$ & $-0,1676(2)$ & $0,1247(1)$ & $0,0539(8)$ \\
N 1 & $0,7753(2)$ & $0,1497(3)$ & $0,1534(1)$ & $0,0402(8)$ \\
C 1 & $0,5978(2)$ & $0,1351(3)$ & $0,1319(2)$ & $0,0433(10)$ \\
C 2 & $0,6865(2)$ & $0,0591(3)$ & $0,1360(2)$ & $0,0390(10)$ \\
C3 & $0,6950(2)$ & $-0,1195(4)$ & $0,1355(2)$ & $0,045(1)$ \\
C4 & $0,8319(2)$ & $0,1865(3)$ & $0,0982(2)$ & $0,0352(9)$ \\
C 5 & $0,4111(2)$ & $0,1867(5)$ & $0,0639(2)$ & $0,064(1)$ \\
C6 & $0,3924(3)$ & $0,3391(5)$ & $0,1101(3)$ & $0,089(2)$ \\
C 7 & $0,4768(3)$ & $0,3894(5)$ & $0,1692(2)$ & $0,078(1)$ \\
C 8 & $0,8049(3)$ & $-0,3401(4)$ & $0,1264(2)$ & $0,073(1)$ \\
C9 & $0,8082(2)$ & $0,1530(3)$ & $0,0100(2)$ & $0,0356(9)$ \\
C 10 & $0,8575(2)$ & $0,0330(3)$ & $-0,0298(2)$ & $0,047(1)$ \\
C 11 & $0,8362(2)$ & $0,0063(4)$ & $-0,1118(2)$ & $0,057(1)$ \\
C 12 & $0,7656(2)$ & $0,0994(4)$ & $-0,1545(2)$ & $0,055(1)$ \\
C 13 & $0,7164(2)$ & $0,2182(4)$ & $-0,1162(2)$ & $0,052(1)$ \\
C 14 & $0,7375(2)$ & $0,2457(4)$ & $-0,0341(2)$ & $0,046(1)$ \\
C 15 & $0,9258(2)$ & $0,2743(3)$ & $0,1232(2)$ & $0,0363(9)$ \\
C 16 & $0,9495(2)$ & $0,3080(3)$ & $0,2049(2)$ & $0,044(1)$ \\
C 17 & $1,0349(2)$ & $0,3929(4)$ & $0,2292(2)$ & $0,054(1)$ \\
C 18 & $1,0984(2)$ & $0,4435(4)$ & $0,1731(2)$ & $0,056(1)$ \\
C 19 & $1,0765(2)$ & $0,4097(4)$ & $0,0929(2)$ & $0,057(1)$ \\
C 20 & $0,9902(2)$ & $0,3263(3)$ & $0,0676(2)$ & $0,046(1)$ \\
\hline
\end{tabular}

$* \mathrm{U}_{\mathrm{eq}}=\frac{1}{3} \sum_{\mathrm{i}} \sum_{\mathrm{j}} \mathrm{U}_{\mathrm{ij}} a_{\mathrm{i}}^{*} a_{\mathrm{j}}^{*} a_{\mathrm{i}} a_{\mathrm{j}}$

* Weitere Einzelheiten zur Kristallstrukturuntersuchung können beim Fachinformationszentrum Karlsruhe, Gesellschaft für wissenschaftlich-technische Information $\mathrm{mbH}$, D-W-7514 Eggenstein-Leopoldshafen 2, unter Angabe der Hinterlegungsnummer CSD56642, der Autoren und des Zeitschriftenzitats angefordert werden. 
[1] S. M. Ali und S. Tanimoto, J. Chem. Soc. Chem. Commun. 1989, 684.

[2] S. M. Ali und S. Tanimoto, J. Org. Chem. 54, 5603 (1989).

[3] M. J. O’Donnell, J. M. Boniece und S. F. Earp, Tetrahedron Lett. 1987, 2641.

[4] M. J. O’Donnell und R. L. Polt, J. Am. Chem. Soc. 47, 2663 (1982).

[5] P. L. Pickard und T. L. Tolbert, Org. Synth. 44, 51 (1964).

[6] M. Brenner und W. Huber, Helv. Chim. Acta 36, 1109 (1953).

[7] K. Hartke und B. Seib, Pharmazie 26, 517 (1970).

[8] A. C. Davis und P. L. Levy, J. Am. Chem. Soc. 73, $3480(1951)$

[9] W.-D. Rudorf und M. Augustin, Z. Chem. 22, 255 (1982).

[10] M. Augustin, W.-D. Rudorf und U. Schmidt, Tetrahedron 32, 3055 (1977).

[11] R. Gompper, Angew. Chem. 74, 251 (1962).

[12] R. Gompper und H. Schäfer, Chem. Ber. 100, 591 (1967)

[13] W.-D. Rudorf, Wiss. Z. Univ. Halle, H. 1, 57 (1983)

[14] L. Dalgaard, H. Kolid-Andersen und S.-O. Lawesson, Tetrahedron 29, 2077 (1973).

[15] W. Dölling, K. Khoudary, M. Augustin, U. Baumeister und $\mathrm{H}$. Hartung, Tetrahedron 44, 4399 (1988).

[16] W. Dölling, K. Khoudary und M. Augustin, J. Prakt. Chem. 331, 573 (1989).

[17] R. Gompper und W. Töpfl, Chem. Ber. 95, 2871 (1962).

[18] SYBYL-Force-Field, Sybyl Molecular Modeling
Software Ver. 5.4 Jan. 1991, Tripos Associates Inc., St. Louis.

[19] E.-U. Würthwein, Tetrahedron Lett. 1983, 581.

[20] A. Schmith und H. J. Lindner, PIMM-Programm, TH Darmstadt, Fachbereich 9.

[21] F. Freeman, D. S. H. L. Kim und J. W. Ziller, Acta Crystallogr. C47, 2124 (1991).

[22] F. Florencio, J. Mohedano und S. Garcia-Blanco, Z. Kristallogr. 180, 131 (1987)

[23] W. J. Adams und L. S. Bartell, J. Mol. Struc. 37(2), 261 (1977)

[24] H. T. Kalff und C. Romers, Acta Crystallogr. 20, 490 (1966).

[25] A. T. McPhail und K. D. Onan, J. Chem. Soc., Perkin Trans. II(9), 1976, 1004.

[26] B. C. Bulman-Page, D. J. Chadwick, M. B. van Niel und D. Westwood, Acta Crystallogr. C 43, 803 (1987).

[27] R. B. Cheik, R. Chaabouni und A. Kallel, Acta Crystallogr. C 48, 283 (1992)

[28] D. Cremer und J. A. Pople, J. Am. Chem. Soc. 97, 1354 (1975).

[29] F. H. Allen, O. Kennard, D. G. Watson, L. Brammer und A. G. Orpen, J. Chem. Soc. Perkin Trans. II, 1987, S 1 .

[30] G. M. Sheldrick, SHELXS 86, Program for Crystal Structure Solution, Univ. Göttingen (1986).

[31] G. M. Sheldrick, SHELX 76, Program for Crystal Structure Determination, Univ. Cambridge (1976).

[32] M. Jaskólski, EDIT, Program to Edit Atomic Information from Atom Data Files, Univ. Poznań (1982).

[33] C. K. Johnson, ORTEP II, Report ORNL-5138. Oak Ridge National Laboratory, Tennessee (1976). 